\title{
Anomalous X-ray scattering studies on semiconducting and metallic glasses
}

\author{
S. Hosokawa ${ }^{1,2, a}$, W.-C. Pilgrim ${ }^{2}$, J.-F. Bérar ${ }^{3}$, and S. Kohara ${ }^{4}$
}

1 Center for Materials Research Using Third-Generation Synchrotron Radiation Facilities, Hiroshima Institute of Technology, Hiroshima 731-5193, Japan

2 Fachbereich Chemie, Physikalische Chemie, Philipps-Universität Marburg, 35032 Marburg, Germany

${ }^{3}$ Institut Néel, Centre National de la Recherche Scientifique/Université Joseph Fourier (CNRS/UJF), 38042 Grenoble Cedex 9, France

${ }^{4}$ Research and Utilization Division, SPring-8/JASRI, Hyogo 679-5198, Japan

Received 13 October 2011 / Received in final form 23 March 2012

Published online 15 June 2012

\begin{abstract}
In order to explore local- and intermediate-range atomic structures of several semiconducting and metallic glasses, anomalous X-ray scattering (AXS) experiments were performed using an improved detecting system suitable for third-generation synchrotron radiation facilities, and the obtained data were analyzed using reverse Monte Carlo (RMC) modelling to obtain partial structure factors and to construct three-dimensional atomic configurations of these glasses. Examples of $\mathrm{GeSe}_{2}$ semiconducting and $\mathrm{Pd}_{40} \mathrm{Ni}_{40} \mathrm{P}_{20}$ metallic glasses are demonstrated to exhibit the feasibility of the combination of AXS and RMC techniques. Importance of an additional combination with neutron scattering is also described for alloys containing light elements.
\end{abstract}

\section{Introduction}

Anomalous X-ray scattering (AXS) is a noble method [1-3] that can provide structural information on both the short- and intermediate-range order (SRO and IRO) around each constituent element in non-crystalline materials. The AXS technique was firstly adopted using X-ray tubes with some different target materials to study partial atomic structures in non-crystalline $\mathrm{GeO}_{2}$ in 1974 by Bondot [4]. Several metallic liquid and amorphous alloys were investigated with the same technique by Waseda and Tamaki $[5,6]$. A significant improvement of the AXS data was achieved by using synchrotron radiation as the X-ray source on amorphous chalcogenide semiconductors, GeSe and $\mathrm{GeSe}_{2}$ [7], and "AXS using synchrotron radiation" was viewed as a promising tool.

Since then, more than three decades have passed. Compared to a related method for structural analysis, X-ray absorption fine structure (XAFS), however, AXS is still rarely used although in contrast to XAFS, it also provides information on the IRO. The cautious progress of this method is related to the difficulties in correcting the

\footnotetext{
a Present address: Department of Physics, Graduate School of Science and Technology, Kumamoto University, Kumamoto 860-8555, Japan, e-mail: hosokawa@sci . kumamoto-u . ac . jp
} 
raw data for fluorescence and Compton contributions, which are complicated and time-consuming procedures. Also, the experimental determination of the anomalous terms is very difficult.

Although recent developments in third-generation synchrotron radiation sources led to a tremendous increase of incoming flux, this did not help to solve the problems of the above mentioned parasitic scattering contributions. This is related to the X-ray detecting system. Pure Ge solid-state-detectors (SSD) are commonly used for AXS experiments. This type of detector is very sensitive and can collect energy-resolved data of scattered X-rays. However, the energy resolution of these detectors is some hundred $\mathrm{eV}$, and hence too large to discriminate between the elastically scattered intensity and the other spurious contributions. Additionally, these detectors exhibit a long dead time of some $\mu \mathrm{s}$. Thus, high flux incident X-rays from third-generation synchrotron radiation facilities help only little to improve the statistical quality of AXS data.

We have developed a detecting system effective for intense third-generation synchrotron radiation. It consists of a graphite analyzer crystal in combination with a long detector arm $[8,9]$, which gives well-resolved elastic scattering signals with high statistical quality. The experimentally obtained differential structure factors, $\Delta_{i} S(Q)$, were analyzed using reverse Monte Carlo (RMC) modelling [10-12] to obtain partial structure factors, $S_{i j}(Q)$, and the corresponding partial pair distribution functions, $g_{i j}(r)$. The RMC method is a useful tool to construct three-dimensional (3D) structural models of disordered materials using mainly experimental diffraction data [13]. The useful combination of AXS experiments and RMC modelling were already given by Waseda and coworkers $[14,15]$. However, the use of RMC was only to extend the limited $Q$ range and to improve the quality of their AXS measurements.

In this paper, we demonstrate our recently developed AXS technique and the feasibility of the combination with RMC modelling for the typical chalcogenide glass $\mathrm{GeSe}_{2}[16,17]$ and the bulk metallic glass $\mathrm{Pd}_{40} \mathrm{Ni}_{40} \mathrm{P}_{20}[18,19]$ as examples. Importance of an additional combination with neutron scattering is also described for the $\mathrm{Pd}_{40} \mathrm{Ni}_{40} \mathrm{P}_{20}$ alloy.

\section{Principle of anomalous X-ray scattering}

The AXS technique utilizes the anomalous variation of the atomic form factor for a specific element if the incident X-ray energy is chosen to be close to its absorption edge. The complex atomic form factor is given as

$$
f(Q, E)=f_{0}(Q)+f^{\prime}(E)+i f^{\prime \prime}(E)
$$

where $f_{0}$ is the usual energy-independent term, and $f^{\prime}$ and $f^{\prime \prime}$ are the real and imaginary parts of the anomalous term, respectively. In general, $f$ is determined by the $Q$-dependent $f_{0}(Q)$ in a normal X-ray scattering process, and the anomalous terms are negligible. When the incident X-ray energy approaches an absorption edge of one of the constituent elements, however, the energy-dependent $f^{\prime}(E)$ and $f^{\prime \prime}(E)$ become important, causing a change in the scattering pattern, $S(Q) . f^{\prime}(E)$ has a large negative minimum and $f^{\prime \prime}(E)$ shows an abrupt jump near the corresponding absorption edge energy.

Thus, an intensity contrast, $\Delta_{i} I$, between two scattering functions can be obtained for the $i$-th element if they are measured closely below the edge ( $E_{\text {near }}$, typically some $10 \mathrm{eV}$ ) and further below the absorption edge $\left(E_{\mathrm{far}}\right.$, typically some $\left.100 \mathrm{eV}\right)$. This contrast can then be expressed as

$$
\beta_{i} \Delta_{i} I\left(Q, E_{\text {far }}, E_{\text {near }}\right)=\Delta_{i}\left[\left\langle f^{2}\right\rangle-\langle f\rangle^{2}\right]+\Delta_{i}\left[\langle f\rangle^{2}\right] \Delta_{i} S(Q),
$$


where $\beta_{i}$ is a normalization constant, $\Delta_{i}$ [ ] indicates the difference of values in the bracket at the energies of $E_{\text {far }}$ and $E_{\text {near }}$, close to the absorption edge of the $i$-th element, and \langle\rangle represents the chemical average of the atomic form factors.

$\Delta_{i} S(Q)$ in Eq. (2) can be expressed by a linear combination of the Faber-Zimantype partial structure factors, $S_{i j}(Q)$, as

$$
\Delta_{i} S(Q)=\sum_{i=1}^{N} \sum_{j=1}^{N} W_{i j}\left(Q, E_{\mathrm{far}}, E_{\mathrm{near}}\right) S_{i j}(Q)
$$

where the weighting factors, $W_{i j}$, are given by

$$
W_{i j}\left(Q, E_{\text {far }}, E_{\text {near }}\right)=x_{i} x_{j} \frac{\Delta_{i}\left[f_{i} f_{j}\right]}{\Delta_{i}\left[\langle f\rangle^{2}\right]} .
$$

Here $x_{i}$ is the concentration of the $i$-th element. It should be noted that compared to $S(Q), \Delta_{i} S(Q)$ highly enhances the partial contributions from the $i$-th element, and suppresses the other partials.

Information on the IRO can also be gained from neutron diffraction employing isotopic substitution (NDIS), from which partial $S_{i j}(Q)$ functions can in principle be obtained directly. Since the contrasts between measurements of differently substituted samples are generally very large, this technique provides very reliable partial information. However, suitable isotopes are very limited and costly. Moreover, the sample must be replaced for each experiment, and there is no guarantee that all the samples have the same atomic structure since microscopic glassy structures are subject to aging, i.e., they depend on the thermal history, and even more important on the preparation process. In contrast, AXS is used on one and the same sample and only the incident X-ray energy is varied. On the other hand, AXS comprises some disadvantages, which must be carefully considered: 1) Light elements have energetically low lying absorption edges and hence the required low incident energies limit the available $Q$ range. 2) The $f^{\prime}$ and $f^{\prime \prime}$ values depend on the environment of the absorbing atom.

\section{Experimental procedure}

Figure 1(a) shows the schematic diagram of the optics and the detecting system used for the present AXS experiment at the beamline BM02 of the European Synchrotron Radiation Facility (ESRF) in Grenoble, France. X-rays generated by a bending magnet source were monochromatized using a $\mathrm{Si}(111)$ double-crystal monochromator with a sagittal focusing system for the second crystal. The monochromator was located between two cylindrically bent mirrors made of Si coated with Pt. This X-ray optics provides a small incident X-ray beam of $0.1 \mathrm{~mm}$ in height and $0.3 \mathrm{~mm}$ in width, and an energy resolution of about $1 \mathrm{eV}$ at an incident X-ray energy of about $10 \mathrm{keV}$.

The diffraction experiments were performed using a standard $\omega-2 \theta$ diffractometer at two energies (typically -20 or $-30 \mathrm{eV}$, and $-200 \mathrm{eV}$ ) below the $\mathrm{K}$ edge of each element. The energy of the incident X-ray beam was calibrated using the absorption edges of samples during the experiments.

Two experimental requirements must be fulfilled in order to obtain $\Delta_{i} S(Q)$ with high statistical quality: 1) A good energy resolution to discriminate the elastic signal from fluorescence and Compton contributions, and 2) a sufficient number of scattered X-ray photons within a reasonable data collection time. For this, we used a graphite crystal energy-analyzer combined with a long detector arm carrying a 


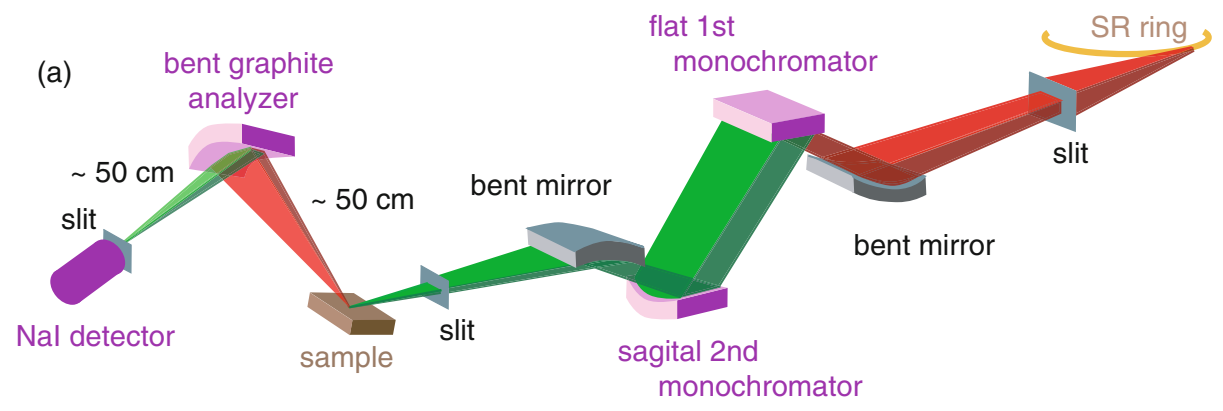

(b)

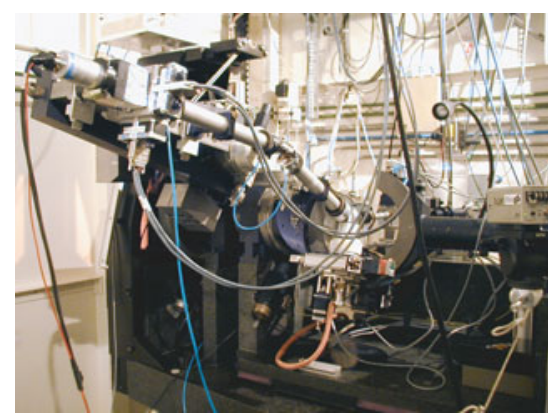

Fig. 1. (a) Schematic diagram and (b) photograph of the AXS setup at BM02/ESRF.

photomultiplier with a NaI crystal scintillator. A photograph of the detecting system is shown in Fig. 1(b).

The graphite crystal provides a good Bragg reflection efficiency of the scattered $\mathrm{X}$-ray photons, while its energy resolution is moderate. The distances from the analyzer crystal to the sample and to the receiving slit in front of the detector were equivalent and long enough (about $50 \mathrm{~cm}$ ) to obtain a good resolution of about $50 \mathrm{eV}$ full-width at half-maximum. Using this detecting system, the elastic signals could mostly be discriminated from fluorescence and Compton contributions. These contributions were estimated from energy scans at constant $Q$ to be less than $0.2 \%$ along the whole experimental $Q$ range. A detailed description of this procedure is given in previous papers $[8,9]$. These energy scans were then used for data correction. Remaining small Compton contributions near the elastic energies were nearly removed in subtracting the two scattering functions at near- and far-edge incident energies. In fact, this reduced the spurious Compton contribution even at high $Q$ values down to less than $0.005 \%$. Regarding the counting rate, curved graphite analyzer crystals with three different radii of curvature were used for different incoming energy ranges. More than three million counts were acquired at the $S(Q)$ maximum, which typically takes less than six hours per scan.

Another advantage of the present detecting system is the much simpler data analysis as compared to the use of SSD. Typically, it takes less than half a day to extract the final $\Delta_{i} S(Q)$ functions out of the raw scattering data. For a similar experiment on $\mathrm{As}_{2} \mathrm{Se}_{3}$ glass [20] where a SSD detector was used, the data analysis took more than two weeks, mainly to correct for the fluorescent X-ray contributions.

\section{Reverse Monte Carlo modelling}

In the RMC simulation technique, atoms of an initial configuration are moved so as to minimize the deviation from experimental structure data, e.g., in these studies, 
Table 1. The $f^{\prime}$ and $f^{\prime \prime}$ values of Ge and Se in electron units at energies measured.

\begin{tabular}{llllll}
\hline Element & Energy $(\mathrm{eV})$ & $f_{\mathrm{Ge}}^{\prime}$ & $f_{\mathrm{Ge}}^{\prime \prime}$ & $f_{\mathrm{Se}}^{\prime}$ & $f_{\mathrm{Se}}^{\prime \prime}$ \\
\hline $\mathrm{Ge}$ & 10903 & -3.647 & 0.510 & -1.750 & 0.656 \\
& 11088 & -7.194 & 0.494 & -1.844 & 0.635 \\
\hline $\mathrm{Se}$ & 12454 & -1.254 & 3.157 & -3.725 & 0.515 \\
& 12639 & -1.113 & 3.084 & -6.310 & 0.500 \\
\hline
\end{tabular}

$S(Q)$ and $\Delta_{i} S(Q)$ functions, using a standard Metropolis Monte Carlo algorithm. In some cases, neutron scattering data needed to be included to determine the atomic arrangement around light elements, where AXS studies are not applicable.

A result of a hard-sphere Monte Carlo simulation of about 5000 atoms was used as the starting configuration. To avoid any physically unrealistic structures, the constraints were applied threefold; shortest atomic distances for all samples, the $8-N$ connectivity rule and the bond angle for semiconducting glasses. Covalently bonded materials generally follow the " $8-N$ rule", which determines the coordination number where $N$ is the number of outer shell electrons.

RMC simulations were then performed on these systems using the RMC ++ program package coded by Gereben et al. [12] with different weighted structure factors. The box length was chosen to correspond to the respective number density.

\section{Examples}

\section{1 $\mathrm{GeSe}_{2}$ semiconducting glass}

$\mathrm{GeSe}_{2}$ semiconducting glass is one of the most typical chalcogenide glasses, which shows a good glass forming ability, and can be applied to infrared optical fibers. The local- and intermediate-range structure of $\mathrm{GeSe}_{2}$ glass was the first target of "AXS using synchrotron radiation" [7]. Later, this system was carefully investigated by Petri et al. [21] using neutron diffraction employing the isotopic substitution (NDIS) technique.

The incident X-ray energies for the present AXS measurements and the $f^{\prime}$ and $f^{\prime \prime}$ values used for the analysis are listed in Table 1. Most of the values are based on calculations by Sasaki [22]. However, corrections were made for $f_{\mathrm{Ge}}^{\prime}$ and $f_{\mathrm{Se}}^{\prime}$ near the absorption edges so as to give reasonable total coordination numbers corresponding to the $8-N$ rule around Ge and Se (4 and 2, respectively) $[16,17]$.

Circles in Fig. 2 show (a) $\Delta_{\mathrm{Ge}} S(Q)$ and (b) $\Delta_{\mathrm{Se}} S(Q)$ obtained from AXS, together with (c) $S(Q)$ measured at an X-ray energy of $12.454 \mathrm{keV}$. As is clearly seen in the figure, considerable contrasts are observed between the three structure factors. $\Delta_{\mathrm{Ge}} S(Q)$ shows a large and sharp prepeak at about $10 \mathrm{~nm}^{-1}$. Moreover, there is a large and sharp negative minimum at $Q=20.5 \mathrm{~nm}^{-1}$. Additionally, the second peak in $\Delta_{\mathrm{Ge}} S(Q)$ at about $Q=35 \mathrm{~nm}^{-1}$ has a shoulder at about $25 \mathrm{~nm}^{-1}$.

On the contrary, $\Delta_{\mathrm{Se}} S(Q)$ shows even a minimum at the prepeak position in $S(Q)$ and $\Delta_{\mathrm{Ge}} S(Q)$. Also, the first peak is very high. Beyond about $30 \mathrm{~nm}^{-1}, \Delta_{\mathrm{Se}} S(Q)$ closely resembles $S(Q)$.

The solid curves in Fig. 2 show the corresponding functions calculated from $S_{i j}(Q)$ obtained from NDIS technique [21] taking into account atomic form factors for X-ray scattering. The agreements between the present AXS and the previous NDIS results are quite good concerning the features and amplitudes except in the prepeak range around $10 \mathrm{~nm}^{-1}$. 


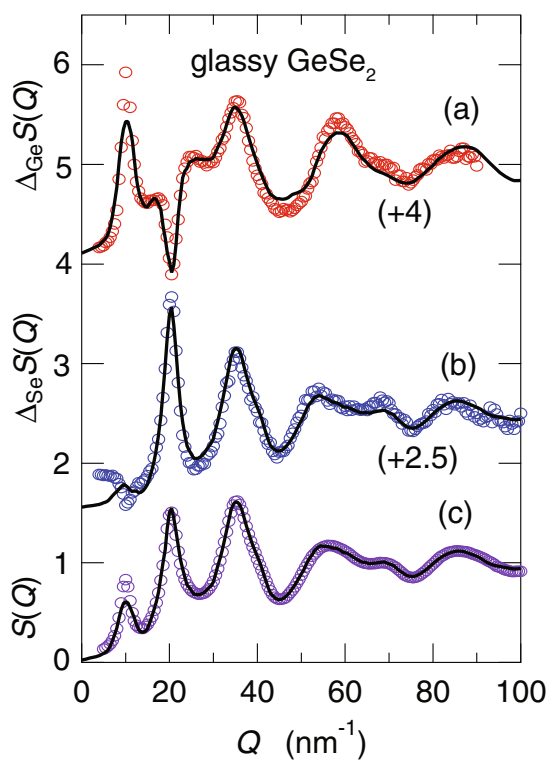

Fig. 2. Circles represent (a) $\Delta_{\mathrm{Ge}} S(Q)$, (b) $\Delta_{\mathrm{Se}} S(Q)$, and $S(Q)$, and the solid curves indicate the corresponding functions obtained from the X-ray weighted neutron data [21]. For clarity, the spectra are displaced upwards. After Ref. [16].

Table 2. Weighting factors of $S_{i j}(Q)$ at the first peak $Q$ position in $S(Q)$.

\begin{tabular}{llll}
\hline data & Ge-Ge & Ge-Se & Se-Se \\
\hline$S(Q)$ & 0.103 & 0.436 & 0.461 \\
$\Delta_{\mathrm{Ge}} S(Q)$ & 0.270 & 0.703 & 0.027 \\
$\Delta_{\mathrm{Se}} S(Q)$ & -0.008 & 0.347 & 0.661 \\
\hline
\end{tabular}

Petri et al. [21] presented two $S_{i j}(Q)$; one shows the raw data with higher prepeaks in $S_{\mathrm{GeGe}}(Q)$ and $S_{\mathrm{GeSe}}(Q)$, and another is the back-Fourier transforms of $g_{i j}(r)$ with smaller prepeaks. The latter data set was used for this calculation. Using the former $S_{i j}(Q)$ data, the agreement in prepeak height becomes better, whereas the errors in $\Delta_{i} S(Q)$ data calculated from the NDIS become larger. For $\Delta_{\mathrm{Se}} S(Q)$, the NDIS results show a small peak at the prepeak position in $S(Q)$. The discrepancy may be due to errors in the AXS data, but the reason is still unclear.

For the RMC modelling of $\mathrm{GeSe}_{2}$ glass, the cut-off values were determined to be $0.22,0.23$, and $0.22 \mathrm{~nm}$ for Ge-Ge, Ge-Se, and Se-Se atomic pairs, respectively, by taking into account the results of $g_{i j}(r)$ functions of $\mathrm{GeSe}_{2}$ obtained by the NDIS technique [21]. The connectivity constraints were adopted preferring the $8-N$ rule, i.e., all Ge atoms were likely coordinated to four Se atoms, and all Se atoms to two Ge atoms. A weak constraint was applied to the Se-Ge-Se bond angle to avoid unphysically large distortions of the $\mathrm{Ge}\left(\mathrm{Se}_{1 / 2}\right)_{4}$ tetrahedra.

Table 2 shows $W_{i j}$ obtained for the different structure factors at the $Q$ position of the first peak in $S(Q)$. As seen in the table, the $W_{i j}$ values are very different from each other, and the $S_{i j}(Q)$ functions can be obtained using these contrasts with the help of RMC modelling.

The thick solid curves in Fig. 3(a) show $S_{\mathrm{GeGe}}(Q), S_{\mathrm{SeSe}}(Q)$, and $S_{\mathrm{GeSe}}(Q)$ obtained from AXS with RMC modelling. A sharp prepeak is seen in $S_{\mathrm{GeGe}}(Q)$ at 
(a)

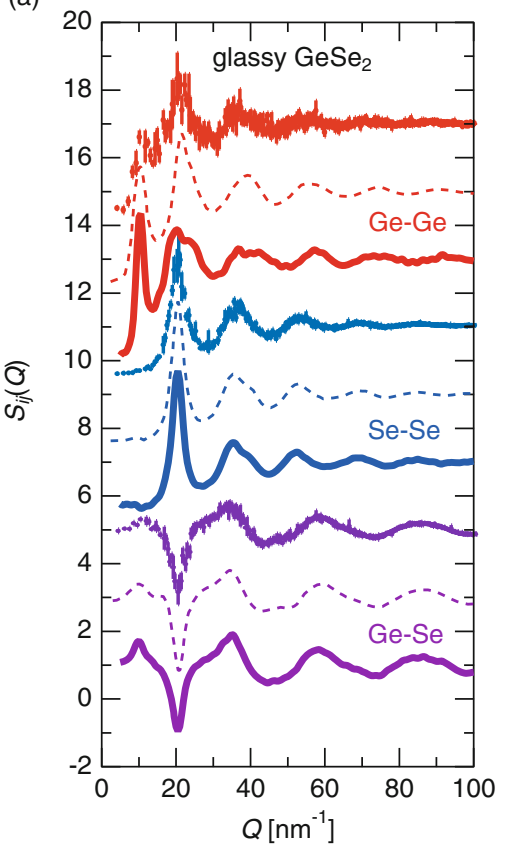

(b)

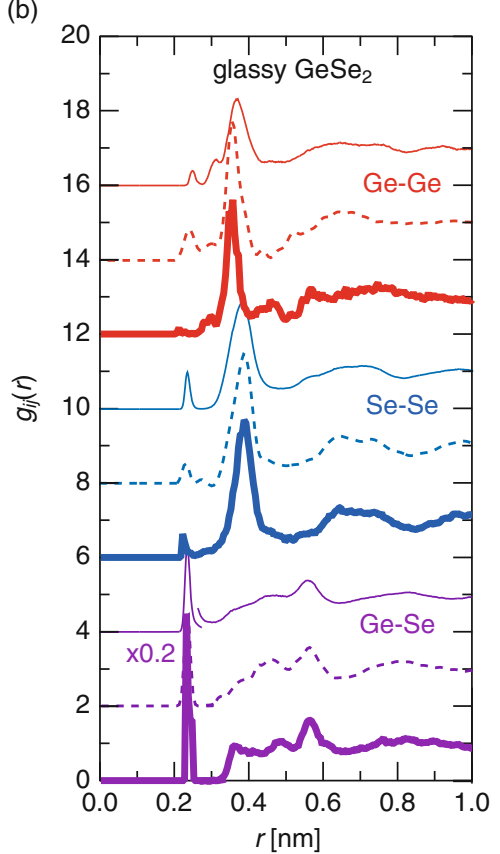

Fig. 3. From top to bottom, (a) $S_{\mathrm{GeGe}}(Q), S_{\mathrm{SeSe}}(Q)$, and $S_{\mathrm{GeSe}}(Q)$, and (b) $g_{\mathrm{GeGe}}(r)$, $g_{\mathrm{SeSe}}(r)$, and $g_{\mathrm{GeSe}}(r)$. Comparison of the present AXS and RMC results (thick solid curves) to results of the NDIS [21] (dashed curves) and the ab initio MD simulation [23] ((a) dots with error bars or (b) thin solid curve). The first peak regions of the $g_{\mathrm{GeSe}}(r)$ functions are reduced by 0.2 in the vertical axis. After Ref. [16]. For clarity, the spectra are displaced upwards by 2 .

about $10 \mathrm{~nm}^{-1}$ with a height of about 2.5. In $S_{\mathrm{GeSe}}(Q)$, a small peak is observed at the about $10 \mathrm{~nm}^{-1}$, and a steep and negative hump is seen at about $20 \mathrm{~nm}^{-1}$. In $S_{\text {SeSe }}(Q)$, there is no indication for a peak at the prepeak position in the other $S_{i j}(Q)$ s, or even a small hump is seen. The first peak has a large height of about 3.5, and subsequent oscillations show large amplitudes, indicating highly ordered atomic correlations.

The dashed curves and dots with error bars indicate $S_{i j}(Q)$ data obtained from NDIS by Petri et al. [21] and an ab initio MD simulation by Massobrio and Pasquarello [23], respectively. Overall features of the spectra exhibit good agreement, in particular for $S_{\mathrm{SeSe}}(Q)$ and $S_{\mathrm{GeSe}}(Q)$.

The $W_{\mathrm{GeGe}}$ values are smaller than the other two correlations due to the small Ge concentration. Thus the errors in $S_{\mathrm{GeGe}}(Q)$ are very large. The low Ge concentration also affects the theoretical calculation as is indicated by the error bars in Fig. 3(a). The theoretical pre peak is smaller, which may be due to the limited system size of the MD simulation. The present AXS data, however, agree well with the theory beyond $20 \mathrm{~nm}^{-1}$ rather than the neutron result. In particular, the shape of the second peak at about $37 \mathrm{~nm}^{-1}$ showing a shoulder at the higher $Q$ side is similar, while the oscillations in the NDIS data are much simpler in this $Q$ range.

The thick solid curves in Fig. $3(\mathrm{~b})$ show $g_{\mathrm{GeGe}}(r), g_{\mathrm{GeSe}}(r)$, and $g_{\mathrm{SeSe}}(r)$ obtained from AXS with RMC modelling, together with the results of NDIS [21] and ab initio MD simulation [23] given by the dashed and thin solid curves, respectively. Overall features again exhibit good agreement, in particular for $g_{\mathrm{SeSe}}(r)$ and $g_{\mathrm{GeSe}}(r)$. 
Table 3. The $f^{\prime}$ and $f^{\prime \prime}$ values of $\mathrm{Pd}, \mathrm{Ni}$, and $\mathrm{P}$ in electron units at energies measured.

\begin{tabular}{llllllll}
\hline Element & Energy $(\mathrm{eV})$ & $f_{\mathrm{Pd}}^{\prime}$ & $f_{\mathrm{Pd}}^{\prime \prime}$ & $f_{\mathrm{Ni}}^{\prime}$ & $f_{\mathrm{Ni}}^{\prime \prime}$ & $f_{\mathrm{P}}^{\prime}$ & $f_{\mathrm{P}}^{\prime \prime}$ \\
\hline $\mathrm{Pd}$ & 24320 & -5.952 & 0.552 & 0.237 & 0.610 & 0.044 & 0.048 \\
& 24150 & -4.228 & 0.559 & 0.239 & 0.618 & 0.045 & 0.049 \\
\hline $\mathrm{Ni}$ & 8313 & -0.073 & 3.723 & -5.706 & 0.480 & 0.274 & 0.408 \\
& 8133 & -0.062 & 3.864 & -3.403 & 0.500 & 0.280 & 0.425 \\
\hline
\end{tabular}

In $g_{\mathrm{GeGe}}(r)$, a small peak is observed at $r=0.23 \mathrm{~nm}$, indicating so-called wrong homopolar bonds, which are not observed in the $\mathrm{GeSe}_{2}$ crystal. Petri et al. [21] and Salmon and Petri [24] gave detailed discussion of these wrong bonds based on their NDIS results. Since such a sharp peak sometimes appears near the cut-off distance in the RMC analysis, however, the existence of wrong bonds cannot reliably be identified from the present RMC calculation.

In this study, we have demonstrated the feasibility of the AXS technique with high statistical quality in combination with RMC modelling. This becomes apparent by comparing to previous $\mathrm{RMC}$ works on the $\mathrm{GeSe}_{2}$ glass [25-27], where only the total $S(Q)$ data were used for the analysis. More detailed discussion on the intermediaterange atomic configurations in $\mathrm{GeSe}_{2}$ semiconducting glass, such as bond angle distributions and connections of the $\mathrm{Ge}\left(\mathrm{Se}_{1 / 2}\right)_{4}$ tetrahedra, is given elsewhere $[16,17]$.

\section{2 $\mathrm{Pd}_{40} \mathrm{Ni}_{40} \mathrm{P}_{20}$ bulk metallic glass}

$\mathrm{Pd}_{40} \mathrm{Ni}_{40} \mathrm{P}_{20}$ glass is a historical bulk metallic glass (BMG), the first one which could be manufactured by simple water quenching $[28,29]$. Later, many BMG materials were discovered [30]. Investigations on the atomic structure of $\mathrm{Pd}_{40} \mathrm{Ni}_{40} \mathrm{P}_{20}$ BMG were performed using AXS technique by Egami et al. [31] and Park et al. [32], and structural models of clusters were proposed based on structures of the approximant crystals.

For a detailed discussion, it is necessary to obtain information on the local environment around the $\mathrm{P}$ atoms from neutron diffraction (ND). This is because the $\mathrm{P}$ atoms have an X-ray scattering cross-section much smaller than that of $\mathrm{Pd}$ and $\mathrm{Ni}$, and the absorption edge of the $\mathrm{P}$ element is too low in energy to carry out AXS. On the other hand, $\mathrm{P}$ has a relatively large neutron scattering cross-section, helping to clarify the atomic positions of $\mathrm{P}$ atoms in the glass. Thus, we have carried out AXS, $\mathrm{XD}$, and ND experiments on $\mathrm{Pd}_{40} \mathrm{Ni}_{40} \mathrm{P}_{20}$ glass. The ND experiment was performed at D4/ILL, and experimental details will be given elsewhere [19]. The incident Xray energies for the AXS measurements and the $f^{\prime}$ and $f^{\prime \prime}$ values used for the data analysis are tabulated in Table 3. The $f^{\prime}$ and $f^{\prime \prime}$ are the theoretical results by Sasaki [22].

Circles in Fig. 4 show experimental data of $\Delta_{\mathrm{Pd}} S(Q)$ and $\Delta_{\mathrm{Ni}} S(Q)$ by AXS, $S(Q) \mathrm{s}$ by XD and ND $\left(S_{X}(Q)\right.$ and $S_{N}(Q)$, respectively). $S(Q)$ was measured at the X-ray energy of $24.150 \mathrm{keV}$. The $Q$ range in the figure is up to $100 \mathrm{~nm}^{-1}$. However, those measured by XD (also $\Delta_{\mathrm{Pd}} S(Q)$ ) and ND are up to 180 and $236 \mathrm{~nm}^{-1}$, respectively. As is seen in the figure, all structure factors resemble each other. However, interesting features were observed from a detailed inspection of these data. The position of the first peak in $\Delta_{\mathrm{Ni}} S(Q)$ shifts to higher $Q$ by about $1.5 \mathrm{~nm}^{-1}$ as compared to $\Delta_{\mathrm{Pd}} S(Q)$. A broad prepeak is seen at about $15 \mathrm{~nm}^{-1}$ in $\Delta_{\mathrm{Ni}} S(Q)$, where a slight enhancement is observed in $S_{N}(Q)$. The second- and third maxima in $S_{N}(Q)$ are shifted towards higher $Q$ with respect to the $S_{X}(Q)$ data. All of these differences result from the different weighting factors of the different experimental methods. 


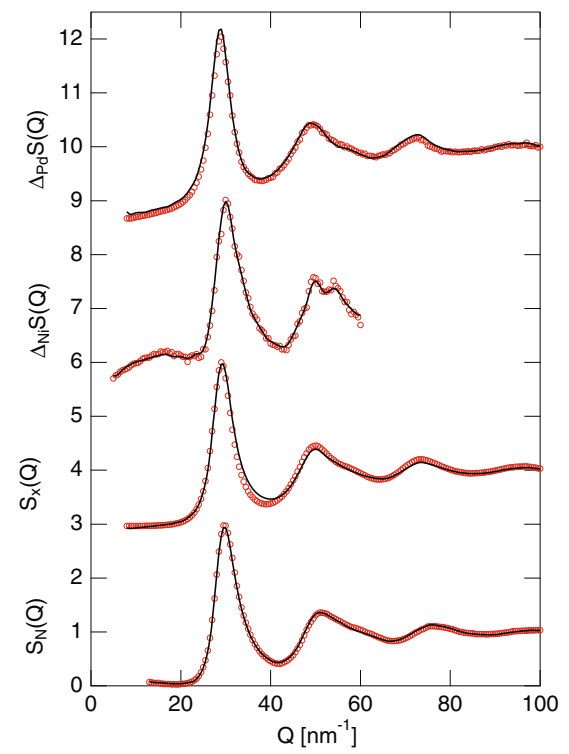

Fig. 4. From top to bottom, circles denote experimental data of $\Delta_{\mathrm{Pd}} S(Q)$ and $\Delta_{\mathrm{Ni}} S(Q)$ by AXS, $S(Q)$ s by XD and ND. The solid curves represent the corresponding fit functions from RMC modelling. For clarity, the spectra are displaced upwards by 3 .

Table 4. Weighting factors of $S_{i j}(Q)$ at the position of the first peak in $S(Q)$.

\begin{tabular}{lllllll}
\hline data & Pd-Pd & Pd-Ni & Pd-P & Ni-Ni & Ni-P & P-P \\
\hline$S_{X}(Q)$ & 0.296 & 0.404 & 0.092 & 0.138 & 0.063 & 0.007 \\
$\Delta_{\mathrm{Pd}} S(Q)$ & 0.537 & 0.377 & 0.086 & 0.000 & 0.000 & 0.000 \\
$\Delta_{\mathrm{Ni}} S(Q)$ & 0.007 & 0.616 & 0.002 & 0.288 & 0.087 & 0.000 \\
$S_{N}(Q)$ & 0.099 & 0.345 & 0.086 & 0.301 & 0.150 & 0.019 \\
\hline
\end{tabular}

Solid curves in Fig. 4 show the corresponding functions obtained from RMC modelling. The cut-off lengths were chosen to be $0.20 \mathrm{~nm}$ between the metallic elements, $\mathrm{Pd}$ and $\mathrm{Ni}$, and $0.18 \mathrm{~nm}$ for the P-related partials. All of the curves coincide well with the respective experimental data. The obtained $g_{i j}(r)$ functions are given in Fig. 5, and the corresponding $S_{i j}(Q)$ functions are given elsewhere [19].

As mentioned above, in order to reliably clarify the local environment around each constituent element, the experimental data sets must comprise sufficient contrast between the weighting factors, $W_{i j}$, of each $S_{i j}(Q)$ function. Table 4 shows $W_{i j}$ obtained for the different structure factors at the $Q$ position of the first peak in $S(Q)$. As is clearly seen in the table, the X-ray related $W_{\mathrm{PP}}$ values are less than $1 \%$. Although $W_{\mathrm{PP}}$ for $S_{N}(Q)$ is also a small value of about $2 \%$, it nevertheless gives a reliable $S_{\mathrm{PP}}(Q)$ function. Similarity is the $W_{\mathrm{NiP}}$ value for $S_{N}(Q)$, about twice as large as that for $S_{X}(Q)$ and $\Delta_{\mathrm{Ni}} S(Q)$.

In order to confirm the importance of the use of ND data, the RMC calculations were performed using only XD data, XD + ND data, and XD + AXS data employing the same initial configuration, constraints of cutoff length, and simulation duration of two days as those used for the simulation with AXS + XD + ND data sets. The results are given in Fig. 5 by dashed curves (XD only), chain curves (XD $+\mathrm{AXS}$ ), and thin solid curves $(\mathrm{XD}+\mathrm{ND})$ together with the present full data set results (thick solid curves). 


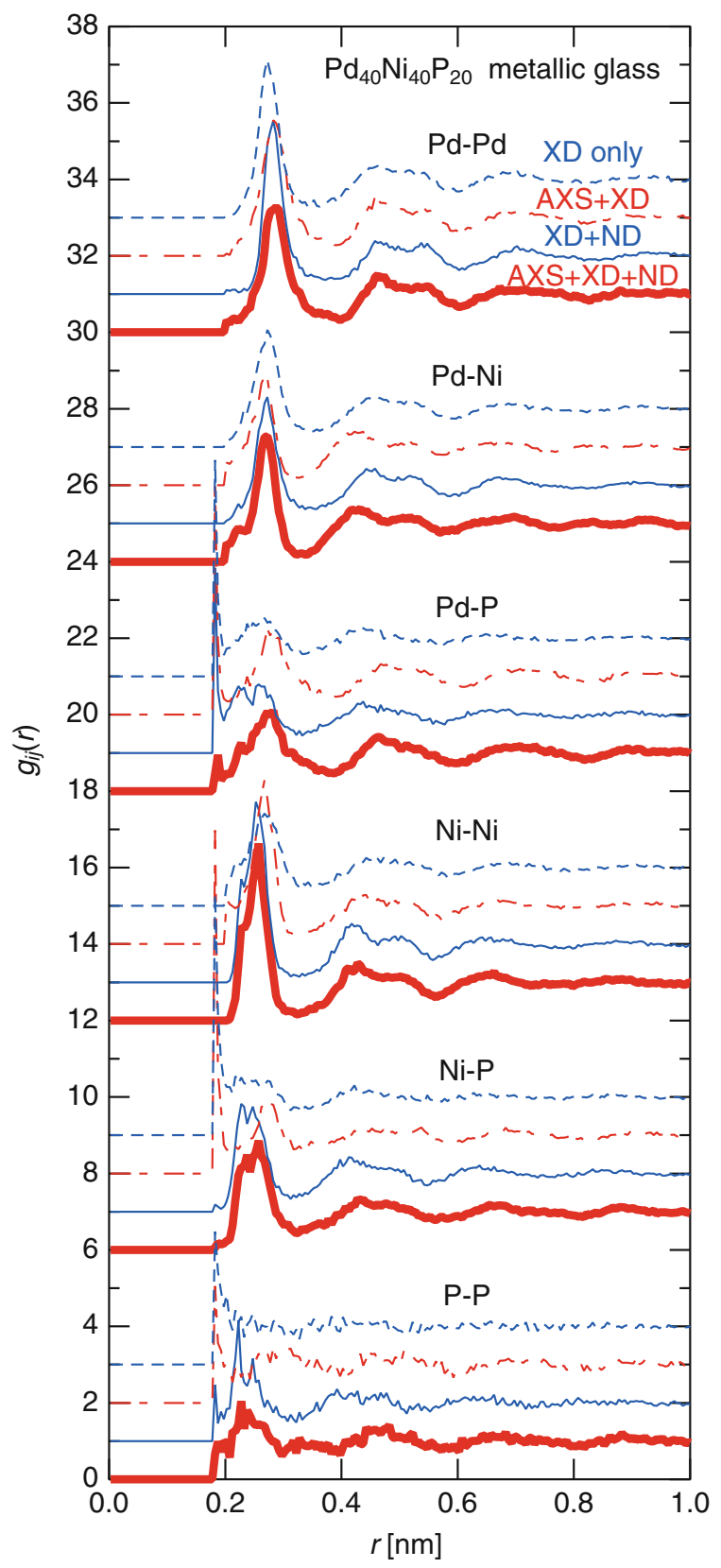

Fig. 5. Comparison of $g_{i j}(r)$ functions obtained from RMC modelling using XD only (dashed curves), AXS + XD (chain curves), XD + ND (thin solid curves), and the present AXS + XD + ND (thick solid curves). For clarity, the spectra are displaced upwards by 1.

The $g_{\mathrm{PP}}(r)$ function obtained from XD only has an enhancement near the cut-off length and shows subsequently no oscillations. This feature is almost the same as the initial spectrum resulting from the hard-sphere random configuration. This result shows that motions of $\mathrm{P}$ atoms in the RMC calculation do not affect the fits to the XD data at all. In addition, the first peak position of $g_{\mathrm{PdPd}}(r)$ is shifted towards smaller $r$ values although $W_{\mathrm{PdPd}}$ in $S_{X}(Q)$ is a large value of about $30 \%$, and the first peak 


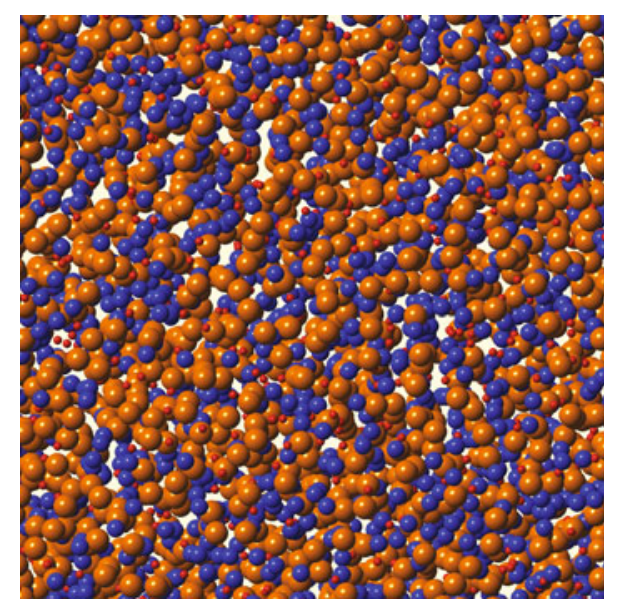

Fig. 6. $3 \mathrm{D}$ atomic configuration of $\mathrm{Pd}_{40} \mathrm{Ni}_{40} \mathrm{P}_{20}$ metallic glass obtained from the RMC modelling. Large, medium, and small balls indicate $\mathrm{Pd}, \mathrm{Ni}$, and $\mathrm{P}$ atoms, respectively.

of $g_{\mathrm{NiNi}}(r)$ is broad. These results indicate that $\mathrm{Ni}$ atoms cannot be discriminated from $\mathrm{Pd}$ atoms if only the XD data were used for the simulation.

By adding the AXS data to the RMC modelling (AXS + XD), clear improvements are seen in the $\mathrm{Pd}-\mathrm{Pd}$ and $\mathrm{Ni}-\mathrm{Ni}$ correlations. However, the P-containing $g_{i j}(r)$ functions are not improved, in particular $g_{\mathrm{PP}}(r)$, due to the small contributions of $\mathrm{P}$ atoms to the X-ray scattering. By adding the ND data to the RMC modelling (XD + ND), most of the first peaks in $g_{i j}(r)$ clearly vary. However, the intermediate-rage structures, such as the second peak positions and the peak features, are very different from the present results where the AXS data are also included (AXS + XD + ND).

From an AXS experiment close to the Ni $K$ edge, Park et al. [32] discussed intermediate-range structures in $\mathrm{Pd}_{40} \mathrm{Ni}_{40} \mathrm{P}_{20}$ metallic glass. They used a cluster model of trigonal prism capped with three octahedra around the $\mathrm{P}$ atom [33]. This suggestion was based on the corresponding crystalline structure. From fitting this model to the experimental $Q S(Q)$ and $Q \Delta_{\mathrm{Ni}} S(Q)$ functions, structural parameters of the glass were calculated. From the present RMC modelling, we could obtain more detailed structural information, which was not included in the model by Park et al. [32]. 1) Pd has an atomic radius larger than the other elements as was already suggested in previous papers [34,35], where this information was obtained by comparing the Pd-Pd $(0.288 \mathrm{~nm})$ and Ni-Ni $(0.256 \mathrm{~nm})$ bond lengths. 2) The P atoms seem to have two interatomic distances to $\mathrm{Pd}$ or $\mathrm{Ni}$ atoms. The Ni-Ni correlation may also have two interatomic sites.

Figure 6 shows a 3D atomic configuration obtained from our RMC modelling. Large, medium, and small balls indicate $\mathrm{Pd}, \mathrm{Ni}$, and $\mathrm{P}$ atoms, respectively. At a glance, a slight tendency for phase separation or clustering is observed as the $\mathrm{Pd}-$ and Ni-rich regions in the figure. To confirm this result, anomalous small angle X-ray scattering would be a powerful tool.

It is believed that the existence of icosahedra avoids crystallization of the metallic glasses [36]. In order to identify this special configuration, a Voronoi analysis was performed for the obtained atomic configuration. A Volonoi polyhedron is denoted by a set of indices $\left(n_{3}, n_{4}, n_{5}, n_{6}\right)$, where $n$ is the number of faces and the number of vertices is indicated by the subscript. The perfect icosahedron is thus described by $\left(\begin{array}{llll}0 & 0 & 12 & 0\end{array}\right)$. The portion of perfect icosahedra is $0.2 \%$ around the $\mathrm{Pd}$ atoms, $6.1 \%$ around the $\mathrm{Ni}$ atoms, and $0.0 \%$ around the $\mathrm{P}$ atoms, indicating that most of icosahedral configurations are observed around the Ni atoms. Although slightly deformed 
icosahedra are seen around the $\mathrm{Pd}$ and $\mathrm{P}$ atoms, they are also observed around the $\mathrm{Ni}$ atoms. A more detailed discussion on the atomic configurations will be given elsewhere [19].

\section{Concluding remarks and future perspective}

AXS experiments on $\mathrm{GeSe}_{2}$ semiconducting and $\mathrm{Pd}_{40} \mathrm{Ni}_{40} \mathrm{P}_{20}$ metallic glasses were performed using an improved detecting system suitable for third-generation synchrotron radiation facilities, and the obtained data were analyzed using RMC modelling to obtain the $S_{i j}(Q)$ s and $g_{i j}(r)$ s, and to construct 3D atomic configurations of these glasses. Importance of an additional combination with neutron scattering is also described for alloys containing light elements.

Our results on semiconducting and metallic glasses clearly demonstrate that AXS in combination with RMC is capable to become a standard method to investigate SRO and IRO of non-crystalline materials. The X-ray source at BM02 of the ESRF is a bending magnet. Other synchrotron X-ray beamlines using insertion devices, such as wigglers or undulators, produce higher fluxes of at least two orders of magnitude. Since the blind time of the present detecting system is very short, and thus, much better statistical quality of AXS data can be expected using such devices as the X-ray source. Experiments on surfaces of glass or liquid are next targets to utilize intenser X-ray sources. Moreover, time-resolved AXS on, e.g., phase-change materials, can be realized.

Finally, the present status and future perspective on RMC simulation are described briefly. RMC technique has widely been applied to model the structure of liquid and amorphous materials, and recently to reveal disorder in crystalline phases [37]. It is known that RMC produces the most disordered structure [38,39], which is consistent with a given set of diffraction/extended X-ray absorption fine structure (EXAFS) data and geometrical constraints. To improve the reliability of the RMC model, we used AXS data, which is sufficiently effective to derive atomic correlations of lighter elements and low concentration elements in alloys up to the intermediaterange scale. The use of RMC is now extended to combine with density functional theory (DFT) [40], DFT/MD simulation [41], and molecular mechanics simulation [42]. Furthermore, recently developed RMC code can handle synchrotron spectroscopic measurement data [43]. Thus, RMC will continue to be a powerful tool to uncover the relationship between unique functional features and hidden structural features in disordered materials on the basis of 3D atomic configurations.

The work on $\mathrm{GeSe}_{2}$ semiconducting glass was performed in collaboration with Prof. I. Oh, Dr. M. Sakurai, and that on PdNiP metallic glass with Dr. D. Szubrin, Dr. A. Zeidler, Dr. H. E. Fischer, Dr. N. Boudet, Prof. T. Ichitsubo, Prof. E. Matsubara, and Dr. N. Nishiyama. SH would like to acknowledge the Japanese Society for Promotion of Science (JSPS) for Grant-in-Aids for Scientific Research for the financial supports for the experiments, and Hiroshima Institute of Technology for the travel expense to the REXS2011 conference site. The AXS experiments were performed at the beamline BM02 in the ESRF (Proposal No. HS1562, HS1860, HS2184, ME1002, and HD162), and the neutron scattering measurements were carried out at the diffractometer D4 in the ILL (Proposal No. 6-05-778).

\section{References}

1. Y. Waseda, The Structure of Non-Crystalline Materials (McGraw-Hill, New York, 1980)

2. Y. Waseda, Novel Application of Anomalous (Resonance) X-ray Scattering for Structural Characterization of Disordered Materials (Springer-Verlag, Heidlberg, 1984) 
3. Y. Waseda, Anomalous X-Ray Scattering for Materials Characterization (SpringerVerlag, Heidlberg, 2002)

4. P. Bondot, Acta Cryst. A 30, 470 (1974)

5. Y. Waseda, S. Tamaki, Philos. Mag. 32, 951 (1975)

6. Y. Waseda, S. Tamaki, Z. Phys. B 23, 315 (1976)

7. P.H. Fuoss, P. Eisenberger, W.K. Warburton, A. Bienenstock, Phys. Rev. Lett. 46, 1537 (1981)

8. S. Hosokawa, Y. Wang, J.-F. Bérar, J. Greif, W.-C. Pilgrim, K. Murase, Z. Phys. Chem. 216, 1219 (2002)

9. S. Hosokawa, J.-F. Bérar, AIP Conf. Proc. 879, 1743 (2007)

10. R.L. McGreevy, L. Pusztai, Molec. Simul. 1, 359 (1988)

11. R.L. McGreevy, M.A. Howe, Ann. Rev. Mater. Sci. 22, 217 (1992)

12. O. Gereben, P. Jóvári, L. Temleitner, L. Pusztai, J. Opt. Adv. Mater. 9, 3021 (2007)

13. M. Maret, F. Lançon, L. Billard, J. Phys.: Condens. Matter 6, 5791 (1994)

14. M. Saito, C. Park, K. Omote, K. Sugiyama, Y. Waseda, J. Phys. Soc. Jpn. 66, 633 (1997)

15. Y. Waseda, S. Kang, K. Sugiyama, M. Kimura, M. Saito, J. Phys.: Condens. Matter 12, A195 (2000)

16. S. Hosokawa, W.-C. Pilgrim, J.-F. Bérar, S. Kohara, Phys. Stat. Sol. (a) 208, 2544 (2011)

17. S. Hosokawa, I. Oh, M. Sakurai, W.-C. Pilgrim, N. Boudet, J.-F. Bérar, S. Kohara, Phys. Rev. B 84, 014201 (2011)

18. S. Hosokawa, H. Sato, T. Ichitsubo, M. Nakatake, N. Happo, J.-F. Bérar, N. Boudet, T. Usuki, W.-C. Pilgrim, E. Matsubara, N. Nishiyama, Phys. Rev. B 80, 174204 (2009)

19. S. Hosokawa, J.-F. Bérar, N. Boudet, S. Kohara, W.-C. Pilgrim, D. Szubrin, A. Zeidler, H. E. Fischer, T. Ichitsubo, E. Matsubara, N. Nishiyama, Phys. Rev. B (submitted)

20. S. Hosokawa, A. Goldbach, M. Boll, F. Hensel, Phys. Stat. Sol. (b) 215, 785 (1999)

21. I. Petri, P.S. Salmon, H.E. Fischer, Phys. Rev. Lett. 84, 2413 (2000)

22. S. Sasaki, KEK Report 1989 (Nat. Lab. High Energy Phys., Tsukuba, 1989), p. 1

23. C. Massobrio, A. Pasquarello, Phys. Rev. B 77, 144207 (2008)

24. P.S. Salmon, I. Petri, J. Phys.: Condens. Matter 15, S1509 (2003)

25. Y. Murakami, T. Usuki, M. Sakurai, S. Kohara, Mater. Sci. Eng. A 449, 544 (2007)

26. Y. Murakami, T. Usuki, S. Kohara, Y. Amo, Y. Kameda, J. Non-Cryst. Solids 353, 2035 (2007)

27. V. Petkov, D. Le Messurier, J. Phys.: Condens. Matter 22, 115402 (2010)

28. H.S. Chen, Mater. Sci. Eng. 23, 151 (1976)

29. A.J. Drehman, A.L. Greer, D. Turnbull, Appl. Phys. Lett. 41, 716 (1982)

30. A. Inoue, Acta Mater. 48, 279 (2000)

31. T. Egami, W. Dmowski, Y. He, R. B. Schwarz, Met. Mater. Trans. 29, 1805 (1998)

32. C. Park, M. Saito, Y. Waseda, N. Nishiyama, A. Inoue, Mater. Trans. JIM 40, 491 (1999)

33. P.H. Gaskel, Acta Metall. 29, 1203 (1981)

34. S. Hosokawa, J.-F. Bérar, N. Boudet, T. Ichitsubo, E. Matsubara, W.-C. Pilgrim, N. Nishiyama, J. Phys.: Conf. Ser. 98, 012013 (2008)

35. S. Hosokawa, J.-F. Bérar, N. Boudet, T. Usuki, T. Ichitsubo, E. Matsubara, N. Nishiyama, J. Phys.: Conf. Ser. 144, 012055 (2009)

36. F.C. Frank, Proc. R. Soc. London, Ser. A 215, 43 (1952)

37. K. Ohara, Y. Kawakita, L. Pusztai, L. Temleitner, S. Kohara, N. Inoue, S. Takeda, J. Phys.: Condens. Matter 22, 404203 (2010)

38. M.T. Dove, M.G. Tucker, D.A. Keen, Eur. J. Mineral. 14, 331 (2002)

39. S. Kohara, S. Kimura, H. Tanaka, N. Yasuda, Y. Fukuyama, H. Murayama, J. Kim, M. Takata, K. Kato, Y. Tanaka, T. Usuki, K. Suzuya, H. Tanaka, Y. Moritomo, T. Matsunaga, R. Kojima, N. Yamada, http://www. epcos.org/papers/pdf _ 2007/paper09_Kohara.pdf 
40. S. Kohara, J. Akola, H. Morita, K. Suzuya, J.K.R. Weber, M.C. Wilding, C.J. Benmore, Proc. Nat. Acad. Sci. USA 105, 7925 (2011)

41. T. Matsunaga, J. Akola, S. Kohara, T. Honma, K. Kobayashi, E. Ikenaga, R.O. Jones,

N. Yamada, M. Takata, R. Kojima, Nature Mater. 10, 129 (2011)

42. H. Morita, S. Kohara, T. Usuki, J. Mol. Liq. 147, 182 (2009)

43. M. Leetmaa, K.T. Wikfeldt, L.G.M. Pettersson, J. Phys.: Condens. Matter 22, 135001 (2010) 